\title{
LASER CHEMISTRY IN SPAIN
}

\author{
by
}

\section{A. GONZÁLEZ UREÑA}

The advent of the laser has effectively created a revolution in many of the modern scientific fields and, in particular, in Chemistry and Physics. In Spain after the chemical laser appeared following its discovery by Pimentel one of his students F. M. G.-Tablas introduced this field to the Autonoma University of Madrid. Spectroscopy and Photochemistry that were well established in different Spanish Universities as well as in the "Consejo Superior de Investigaciones Científicas" developed into the Laser application field during the late seventies and early eighties. By which time one of the pioneering groups that of J. M. Figuera had built several chemical Lasers. Simultaneously the Spanish molecular reaction dynamics community, consisting of both experimentalists and theoreticians, that was growing very rapidly, entered onto the discipline of Laser Chemistry incorporating beam-laser interactions and the dynamics of excited species.

The current spectrum of the Spanish research community involved in Laser Chemistry is composed of approximately a dozen groups including 50 scientists mainly from Photochemistry and Spectroscopy with only a minority belonging to beam and/or theoretical work. Most of the groups are from Madrid (group leaders: Bermejo, Borondo, Delgado-Barrio, Escudero, Figuera, González-Díaz, González Ureña, Tablas, Tabares) and only a few from the rest of Spain (Castaño in Bilbao and Martínez Ataz in Ciudad Real). The success of this small community is based on support from the CICYT (The National Research foundation Agency) although EEC support is slightly increasing. Obviously the field in this Country needs both economical support and international cooperation to guarantee its development and consolidation.

This special issue entitled "Laser Chemistry in Spain" gathers articles from Spanish laboratories and research groups from a large "range" of applications in which the laser plays a decisive role. 